\title{
BER-based Optimal Power Allocation for Cognitive Relay Networks
}

\author{
Jun Cui, Weijia Lei and Xianzhong Xie \\ Chongqing Key Lab of Mobile Communications Technology, \\ Chongqing University of Posts and Telecommunications, Chongqing, China \\ cuijun945@163.com, \{leiwj, xiexz\}@cqupt.edu.cn
}

\begin{abstract}
In this paper, we consider an optimal power allocation scheme for Rayleigh fading channels in cognitive relay networks considering both total transmit power and average interference power constraints. We derive the optimal power allocation scheme between the cognitive source and relay aiming at minimizing the bit error rate of cognitive system by applying convex optimization method. Simulation results indicate that the proposed scheme outperforms average power allocation scheme when the relay is located at different positions.
\end{abstract}

Keywords-cognitive radio; relay technology; power allocation; bit error rate; convex optimization

\section{INTRODUCTION}

In recent years, with the widespread popularity of wireless communication devices and services, spectrum as a non-renewable natural resources has become increasingly scarce. However, the spectrum efficiency of the conventional fixed spectrum allocation scheme is very low. This spurred the search for a new and efficient technology to use the spectrum rationally. Thus, cognitive radio (CR) [1] was proposed for improving spectrum utilization. In CR networks, cognitive users (CU) are permitted to use the licensed band so long as they protect the data transmission of the licensed user (PU) using spectrum overlay and underlay.

Today, the researchers most assume that cognitive transmitter and receiver hold the same spectrum, or use their common part of the available spectrum [1]. However, in the practice, CUs often locate at different coverage of PUs, thus the spectrum availability for CUs varies in different areas of PUs. Reference [2] first proposed a cognitive cooperative relay scheme for cognitive source and destination with different available spectrum. In this paper, we consider a communication scheme for cognitive source and destination having different available spectrum due to locating at different coverage of PUs.

Relayed transmission is a way to attain broader coverage by splitting the communication link from the source to the destination into several shorter links [3]. In this paper, by introducing a cognitive relay between cognitive source and destination, it not only achieves the communication between cognitive source and destination which have different available spectrum, but also improves the performance of the cognitive system.

For handheld terminals, the power is undoubtedly a very important resource. Reference [4] analyzed the Optimal Power Allocation (OPA) based on the physical network coding in cognitive relay network. Currently, there are a lot of papers which study the OPA for cognitive relay networks [4], [5], however, we seldom find a paper aiming at minimizing bit error rate (BER) in cognitive relay networks. In this paper, we give a new OPA scheme between the source and relay aiming at minimizing BER by applying convex optimization methods [6].

The rest of this paper is organized as follows. In section II, we introduce the system model and formulate the power allocation problem. Then, the average power allocation (APA) scheme is given in section III and the OPA scheme is shown in section IV. Simulation results are presented in section $\mathrm{V}$ and this paper is concluded in section VI.

\section{SYSTEM MODEL AND PROBLEM FORMULATION}

\section{A. Channel and System Model}

This paper considers a communication scheme for cognitive source and destination having different available spectrum due to locating at different coverage of PUs [2], as shown in figure.1. We assume that there is a PU $a$ in the spectrum sensing coverage $R_{s}$ of source $s$, similarly, PU $b$ locates in the spectrum sensing coverage $R_{d}$ of destination $d$, and the PU $a$ and $b$ is present. The channel set of PU $a$ and $b$ is represented by $\mathrm{CH}_{a}$ and $\mathrm{CH}_{b}$ respectively, the source and destination can't communicate directly without the same available spectrum. We find a cognitive DF relay $r$ between the source and destination for forwarding data, so the source and destination can communicate each other through relay.

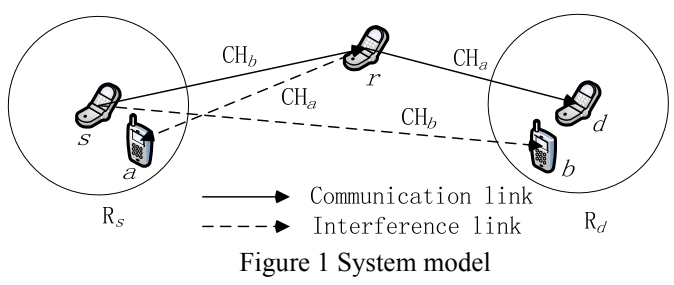

CUs need to control transmit power in order to achieve coexistence with the PUs. A general scheme of power control is discussed, where the transmitter controls its power to satisfy the interference power constraint (IPC) predefined by the PUs [4], [5]. For the IPC, there are two power control methods of average and peak, and we have chosen the average IPC. The average IPC of PU $a$ and $b$ is represented by $Q_{a}$ and $Q_{b}$ respectively. The system modulation scheme is binary differential phase shift keying (2DPSK). The communication process between $s$ and $d$ will have the following steps:

- $s$ transmits data to $r$ with $\mathrm{CH}_{b}$ under the IPC of $Q_{b}$; 
- $\quad r$ receives data from $s$ and decodes it;

- r forwards data to d with $\mathrm{CH}_{a}$ under the IPC of $Q_{a}$;

- $d$ receives data from $r$ and decodes it.

The communication and interference link are assumed to be independent flat Rayleigh fading. Further, we assume that perfect channel state information (CSI) is available at SUs [4]. Channel noise is zero mean additive white Gaussian noise with power spectral density $N_{0}$. The channel gain between any node $i$ and node $j$ with $h_{i j}$ is zero mean with the distribution $\mathrm{CN}\left(0, \Omega_{i j}\right)$ (circularly symmetric complex Gaussian), so the channel power gain with $\left|h_{i j}\right|^{2}$ is exponential distribution with mean [7]

$$
\Omega_{i j}=\left(\frac{c}{4 \pi f_{n} L_{i j}}\right)^{\alpha}
$$

where $L_{i j}$ represents the distance between the node $i$ and $j ; \alpha$ represents the path loss exponent, ranging generally between 2 and 5, and we take 3 in this paper; c is the speed of light; the $f_{n}(n=a, b)$ is the carrier frequency for PU $a$ and $b$.

\section{B. Problem Formulation}

The transmit power of source and relay are represented by $P_{1}$ and $P_{2}$ respectively, and the upper limit of total transmit power is $P_{T}$. The $P_{1}, P_{2}$ is required to meet not only the total power constraint, but also the average IPC set by PU, i.e.

$$
\begin{gathered}
P_{1}+P_{2} \leq P_{T} \\
E\left[P_{1}\left|h_{s b}\right|^{2}\right]=P_{1} \Omega_{s b} \leq Q_{b} \\
E\left[P_{2}\left|h_{r a}\right|^{2}\right]=P_{2} \Omega_{r a} \leq Q_{a}
\end{gathered}
$$

where the symbol of $\mathrm{E}$ in (3) and (4) indicates the statistical average.

The transmit rate between $\mathrm{CU}$ is represented by $R(\mathrm{bit} / \mathrm{s})$, and the average bit signal-to-noise (SNR) $(\mathrm{Eb} / N 0)$ received by relay and destination are represented by $\bar{\gamma}_{1}, \overline{\gamma_{2}}$ respectively,

$$
\begin{gathered}
\overline{\gamma_{1}}=\frac{\Omega_{s r} P_{1}}{N_{0} R}=G_{1} P_{1} \\
\overline{\gamma_{2}}=\frac{\Omega_{r d} P_{2}}{N_{0} R}=G_{2} P_{2}
\end{gathered}
$$

where $\frac{\Omega_{s r}}{N_{0} R}, \frac{\Omega_{r d}}{N_{0} R}$ in (5) and (6) are abbreviated as $G_{1}, G_{2}$ respectively.

There is no direct link between $s$ and $d$. And $r$ acts as a DF relay, then the average BER can be expressed as [3]

$$
\begin{aligned}
P_{e}^{s d} & =P_{e}^{s r}+P_{e}^{r d}-2 P_{e}^{s r} P_{e}^{r d} \approx P_{e}^{s r}+P_{e}^{r d} \\
& =\frac{1}{2}\left[\frac{1}{1+G_{1} P_{1}}+\frac{1}{1+G_{2} P_{2}}\right]
\end{aligned}
$$

where $P_{e}^{s r}$ and $P_{e}^{s r}$ are the average BER from $s$ to $r$ and $r$ to $d$ respectively.
From (7), the OPA based on minimizing BER is equivalent to solving the problem:

$$
\begin{gathered}
\left(P_{1}^{*}, P_{2}^{*}\right)=\arg \min _{P_{1}, P_{2}} \frac{1}{1+G_{1} P_{1}}+\frac{1}{1+G_{2} P_{2}} \\
\text { s.t. }\left\{\begin{array}{l}
0<P_{1}+P_{2} \leq P_{T} \\
0<P_{1} \Omega_{s b} \leq Q_{b} \\
0<P_{2} \Omega_{r a} \leq Q_{a}
\end{array}\right.
\end{gathered}
$$

The objective function (8) is a convex function, and the constraint (9) is a linear function, so the problem is a convex optimization problem. The problem is solvability and has the only solution.

\section{BER-BASED AVERAGE POWER ALLOCATION}

This section discusses a simple APA scheme. We can't simply make $P_{1}=P_{2}=P_{\mathrm{T}} / 2$, because this may not satisfy the IPC. We can take such an approach: firstly, the source and relay is allocated the same power. Secondly, we estimate whether the node meet the IPC: if the two nodes don't exceed the IPC, then we keep silence; if only one node exceeds the IPC, the node is allocated with its threshold value, and the exceeded power is allocated to the other node; if two nodes both exceed the IPC, they are allocated with their thresholds, that is

$$
\left(P_{1}^{*}, P_{2}^{*}\right)= \begin{cases}\left(\frac{P_{T}}{2}, \frac{P_{T}}{2}\right) & \left(P_{T} \leq \min \left\{\frac{2 Q_{b}}{\Omega_{s b}}, \frac{2 Q_{a}}{\Omega_{r a}}\right\}\right) \\ \left(\frac{Q_{b}}{\Omega_{s b}}, P_{T}-\frac{Q_{b}}{\Omega_{s b}}\right) & \left(\frac{2 Q_{b}}{\Omega_{s b}} \leq \frac{2 Q_{a}}{\Omega_{r a}}, \text { 且 } \frac{2 Q_{b}}{\Omega_{s b}}<P_{T} \leq \frac{Q_{a}}{\Omega_{r a}}+\frac{Q_{b}}{\Omega_{s b}}\right) \\ \left(P_{T}-\frac{Q_{a}}{\Omega_{r a}}, \frac{Q_{a}}{\Omega_{r a}}\right) & \left(\frac{2 Q_{a}}{\Omega_{r a}}<\frac{2 Q_{b}}{\Omega_{s b}}, \text { 且 } \frac{2 Q_{a}}{\Omega_{r a}}<P_{T} \leq \frac{Q_{a}}{\Omega_{r a}}+\frac{Q_{b}}{\Omega_{s b}}\right) \\ \left(\frac{Q_{b}}{\Omega_{s b}}, \frac{Q_{a}}{\Omega_{r a}}\right) & \left(P_{T}>\frac{Q_{a}}{\Omega_{r a}}+\frac{Q_{b}}{\Omega_{s b}}\right)\end{cases}
$$

\section{BER-BASED OPTIMAL POWER ALLOCATION}

Through the APA scheme is simple, but in most cases it's not the best. So this section gives an OPA scheme based on minimizing BER. Lagrangian for the problem (8) is given as

$$
\begin{aligned}
J= & \frac{1}{1+G_{1} P_{1}}+\frac{1}{1+G_{2} P_{2}}+\mu_{1}\left(P_{1}+P_{2}-P_{T}\right)+ \\
& \mu_{2}\left(P_{1} \Omega_{s b}-Q_{b}\right)+\mu_{3}\left(P_{2} \Omega_{r a}-Q_{a}\right)
\end{aligned}
$$

where $\mu_{1}, \mu_{2}, \mu_{3}$ are the lagrange multipliers. So the KKT conditions of the problem is

$$
\begin{gathered}
P_{1}>0, P_{2}>0, \mu_{i} \geq 0, i=1,2,3 \\
\mu_{1}\left(P_{1}+P_{2}-P_{T}\right)=0 \\
\mu_{2}\left(P_{1} \Omega_{s b}-Q_{b}\right)=0 \\
\mu_{3}\left(P_{2} \Omega_{r a}-Q_{a}\right)=0 \\
\frac{\partial J}{\partial P_{1}}=-\frac{G_{1}}{\left(1+G_{1} P_{1}\right)^{2}}+\mu_{1}+\mu_{2} \Omega_{s b}=0 \\
\frac{\partial J}{\partial P_{2}}=-\frac{G_{2}}{\left(1+G_{2} P_{2}\right)^{2}}+\mu_{1}+\mu_{3} \Omega_{r a}=0
\end{gathered}
$$


According to the different situations of IPC, we discuss the four situations for the optimal solution of the problem.

\section{A. Case I: Interferences not exceed IPC values}

Here the transmit powers of source and relay do not exceed IPC levels of PUs $b$ and $a$. Based (12a), (12c) and (12d), we can get $\mu_{2}=\mu_{3}=0$. Substituting it into (12e) and (12f), we can obtain

$$
\mu_{1}=\frac{G_{1}}{\left(1+G_{1} P_{1}\right)^{2}}=\frac{G_{2}}{\left(1+G_{2} P_{2}\right)^{2}}
$$

From the above equation, we get $\mu_{1} \neq 0$, and from (12b), there is

$$
P_{1}+P_{2}-P_{T}=0
$$

Associating (12) and (13), we can obtain

$$
\left\{\begin{array}{l}
\frac{G_{1}}{\left(1+G_{1} P_{1}\right)^{2}}=\frac{G_{2}}{\left(1+G_{2} P_{2}\right)^{2}} \\
P_{1}+P_{2}-P_{T}=0
\end{array}\right.
$$

The solution of the above equation is

$$
\left\{\begin{array}{l}
P_{1}^{*}=\frac{P_{T} \sqrt{G_{1} G_{2}}+\left(\sqrt{G_{1} / G_{2}}-1\right)}{G_{1}+\sqrt{G_{1} G_{2}}} \\
P_{2}^{*}=\frac{P_{T} G_{1}-\left(\sqrt{G_{1} / G_{2}}-1\right)}{G_{1}+\sqrt{G_{1} G_{2}}}
\end{array}\right.
$$

Substituting the above equation into (9), we have

$$
\left\{\begin{array}{l}
P_{T} \leq\left[\frac{Q_{b}}{\Omega_{s b}}\left(\sqrt{\frac{G_{1}}{G_{2}}}+1\right)+\frac{1}{\sqrt{G_{1} G_{2}}}-\frac{1}{G_{2}}\right]=P_{T}^{\prime} \\
P_{T} \leq\left[\frac{Q_{a}}{\Omega_{r a}}\left(\sqrt{\frac{G_{2}}{G_{1}}}+1\right)+\frac{1}{\sqrt{G_{1} G_{2}}}-\frac{1}{G_{1}}\right]=P_{T}^{\prime \prime}
\end{array}\right.
$$

where $P_{T}^{\prime}$ and $P_{T}^{\prime \prime}$ are the upper limit of $P_{T}$, the (17) can also be expressed as

$$
P_{T} \leq \min \left\{P_{T}^{\prime}, P_{T}^{\prime \prime}\right\}
$$

\section{Case II: Source power is limited due to IPC of PU}

When $P_{T}>\min \left\{P_{T}^{\prime}, P_{T}^{\prime \prime}\right\}$, if we continue to take the power allocation scheme of case I, there is at least one node between source and relay will exceed the IPC of PU, so we need to find a new power allocation scheme.

The optimal solution under this scenario is given as

$$
\left\{\begin{array}{l}
P_{1}^{*}=\frac{Q_{b}}{\Omega_{s b}} \\
P_{2}^{*}=P_{T}-\frac{Q_{b}}{\Omega_{s b}}
\end{array}\right.
$$

This case occurs should meet the following conditions

$$
\left\{\begin{array}{l}
P_{T}^{\prime}<P_{T}^{\prime \prime} \\
P_{T}^{\prime}<P_{T} \leq \frac{Q_{a}}{\Omega_{r a}}+\frac{Q_{b}}{\Omega_{s b}}
\end{array}\right.
$$

\section{Case III: Relay power is limited due to IPC of PU}

The transmit power of relay is limited with PU $a$ experiencing the maximum possible interference level. The optimal solution under this scenario is given as

$$
\left\{\begin{array}{l}
P_{1}^{*}=P_{T}-\frac{Q_{a}}{\Omega_{r a}} \\
P_{2}^{*}=\frac{Q_{a}}{\Omega_{r a}}
\end{array}\right.
$$

This case occurs should meet the following conditions

$$
\left\{\begin{array}{l}
P_{T}^{\prime \prime}<P_{T}^{\prime} \\
P_{T}^{\prime \prime}<P_{T} \leq \frac{Q_{a}}{\Omega_{r a}}+\frac{Q_{b}}{\Omega_{s b}}
\end{array}\right.
$$

Case IV: Both source and relay power is limited due to IPC of $P U$

When both the SUs transmit powers are limited due to higher interferences at PUs, the optimal solution given as

$$
\left\{\begin{array}{l}
P_{1}^{*}=\frac{Q_{b}}{\Omega_{s b}} \\
P_{2}^{*}=\frac{Q_{a}}{\Omega_{r a}}
\end{array}\right.
$$

Obviously, the total power allocated to them should be less than $P_{T}$, and we can obtain

$$
P_{T}>\frac{Q_{a}}{\Omega_{r a}}+\frac{Q_{b}}{\Omega_{s b}}
$$

\section{SIMULATION RESULTS}

In this section, we present the simulation results for the power distribution effect under the Rayleigh flat fading channel applied the Monte Carlo method, which simulation platform takes Matlab 7.1 and the number of bits for simulation is $10^{6}$. The simulation scenario is shown in Fig. 2. We assume that the sensing range of CUs is 50 meters, and the node $s, d, a$ and $b$ are located at $(-200,0),(200,0),(-181$, $-32)$ and $(225,28)$. Taking into account the BER is related with the location of the relay, three typical positions of the relay are considered, near the source (NS), near the middle point (NM) and near the destination (ND) are located (-148, $-27),(12,2)$ and $(162,24)$. Meter is the unit of distance here.

The carrier frequency of the PU $a$ and $b$ is set to $610 \mathrm{MHz}$ and $600 \mathrm{MHz}$ respectively. The noise power spectral density is $-174 \mathrm{dBm} / \mathrm{Hz}$ [7]; the system spectral efficiency is $1 \mathrm{bps} / \mathrm{Hz}$; the transmit rate between SUs is $R=64 \mathrm{kbit} / \mathrm{s}$; the system bandwidth is $B=64 \mathrm{kHz}$. Further, we assume that the PU $a$ and $b$ have the same IPC, i.e., $Q_{a}=Q_{b}$. 


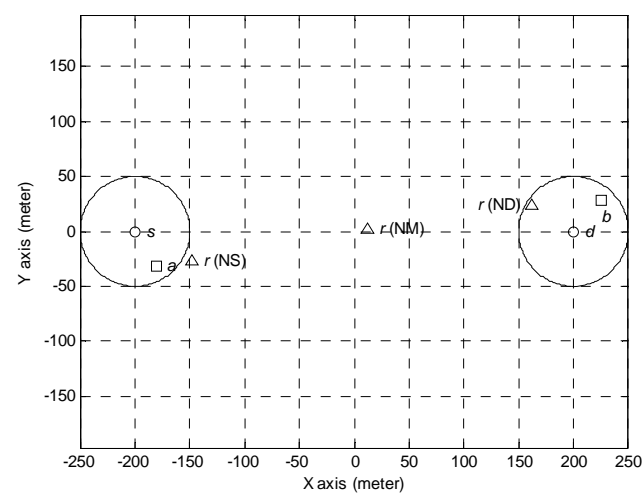

Figure 2 System model in simulation

Fig. 3 compares the BER under different IPC applied APA when relay is located at NM. We can find the BER will no longer be reduced when the transmit power exceeds a certain value. Though the system can provide large power, the power distributed the CUs is limited due to the IPC. In addition, we can find the floor of BER will reduce with increasing of IPC.

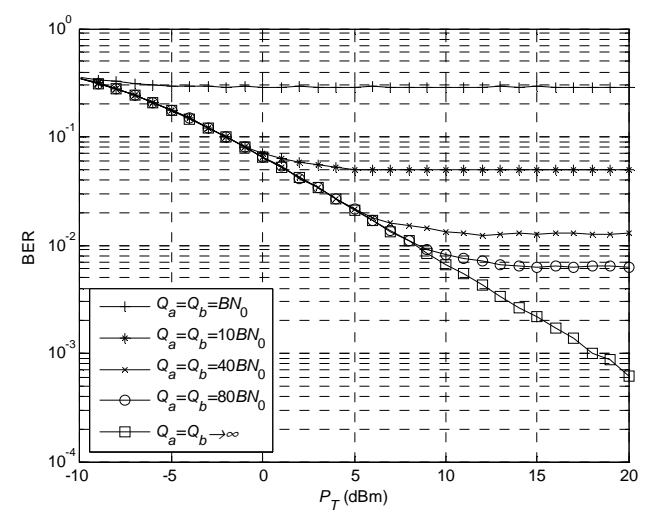

Figure 3 The BER simulation values with different IPC

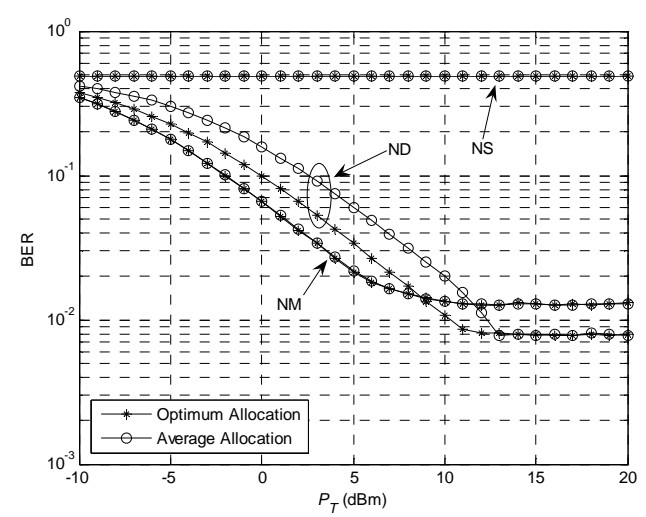

Figure 4 The BER simulation values for OPA and APA

Fig. 4 compares APA and OPA in terms of BER for relay located at different positions, where the IPC is $40 B N_{0}$. From the fig. 4, the BER applied OPA is better than APA for different positions. Under the OPA, the BER for the relay located at NM is smaller when the total transmit power is less than $9 \mathrm{dBm}$; the BER is no longer reduced due to the IPC when the total transmit power is greater than $9 \mathrm{dBm}$, and the BER for the relay located at ND is smaller; the relay located at NS has a smaller transmission power, therefore the BER is large (close to $50 \%$ ) due to the IPC of the PU $a$. From three typical positions of the relay, the BER is large for the relay located at NS; the BER is small when the relay transmit power is limited by IPC for the relay located at NM; the BER is smaller when the relay transmit power is not limited by IPC for the relay located at ND. Therefore, the optimal location of relay dynamically changes with the changing of the total transmit power.

\section{CONCLUSIONS}

We have given a cognitive communication scheme employing relay for cognitive source and destination with different available spectrum situation. Then we have formulated an optimization problem to minimize the BER of the cognitive communication under a total power and average interference power constraints. The optimum power allocation algorithm has been derived by using KKT conditions for different cases. The numerical and simulation results show the BER is limited due to IPCs. The BER is affected by the position of relay and the OPA is better than APA when the relay is located different positions.

\section{ACKNOWLEDGMENT}

This paper was supported by the National Nature Science Foundation of China (61271259), the Chongqing Nature Science Foundation (CTSC2011jjA40006), the Research Project of Chongqing Education Commission (KJ120501, KJ120502), the transformation project of excellent achievement of Chongqing Municipal Education Commission (Kjzh11206).

\section{REFERENCES}

[1] B. B. Wang and K. J. R. Liu, "Advances in cognitive radio networks: A survey," IEEE J. on Select. Topics in Signal Process., vol. 5, no. 1, pp. 523, Feb. 2011

[2] X. W. Gong, W. Yuan, W. Liu, etal., "A Cooperative Relay Scheme for Secondary Communication in Cognitive Radio Networks," in Proc. IEEE Global Telecommun. Conf. (GLOBECOM) 2008, pp. 1-6, 2008.

[3] M. O. Hasna and Mohamed-Slim Alouini, "Performance analysis of two-hop relayed transmissions over Rayleigh fading channels," in Proc. IEEE 56th Veh. Technol. Conf. (VTC) 2002, pp. 1992-1996, 2002.

[4] L. K. S. Jayasinghe, N. Rajatheva and M. Latva-aho, "Optimal Power Allocation for PNC Relay Based Communications in Cognitive Radi-o," in Proc. IEEE Int. Conf. on Commun. (ICC) 2011, pp. 1-5, 2011.

[5] X. Kang, Ying-Chang Liang, A. Nallanathan, etal. , "Optimal Power Allocation for Fading Channels in Cognitive Radio Networks: Ergodic Capacity and Outage Capacity," IEEE Trans. on Wireless Commun., vol. 8, no. 2, pp. 940- 950, Feb. 2009

[6] S. Boyd and L. Vandenberghe, Convex Optimization. Cambridge, UK: Cambridge University Press, 2004.

[7] W. Su, J. D. Matyjas and S. Batalama, "Active Cooperation Between Primary Users and Cognitive Radio Users in Heterogeneous Ad-Hoc Networks", IEEE Trans. on Signal Process., vol. 60, no. 4, pp. 1796-1805, APR. 2012. 